\section{The role of bedside ultrasound in the diagnosis and outcome of patients with acute respiratory failure}

\author{
Andrea Bellone, ${ }^{1}$ Massimiliano Etteri, ${ }^{1}$ \\ Carlo Maino, ${ }^{2}$ Chiara Bonetti, ${ }^{2}$ \\ Anna Natalizi ${ }^{2}$ \\ 'Emergency Department, Sant'Anna \\ Hospital, San Fermo della Battaglia; \\ ${ }^{2}$ Emergency Department, Valduce \\ Hospital, Como, Italy
}

becoming a standard tool for a rapid diagnosis and treatment of acute respiratory failure. ${ }^{7,8} \mathrm{~A}$ recent study demonstrated a high concordance between radiography and ultrasonography and the latter proved to be more accurate in distinguishing free pleural effusion. ${ }^{9}$ Moreover, Reissig et al. evaluated the role of emergency ultrasound of the chest and they stated that sonography allows immediate diagnosis of pulmonary embolism, pneumothorax, pneumonia, pleural effusion and provides a basis for further treatment-related decisions. ${ }^{10}$ The aim of our observational study was to compare the outcome of patients admitted to the hospital with acute respiratory failure according to a preliminary approach, the bedside ultrasound evaluation.

\section{Abstract}

The aim of the present study was to evaluate the relationship between a bedside ultrasound evaluation during an episode of acute respiratory failure and the patient's outcome. A retrospective observational study was conducted in the emergency departments (EDs) of two hospitals in Como (Sant'Anna Hospital and Valduce Hospital) over two years. Two hundred and twenty eight adult patients with acute respiratory failure were recruited for the study. One hundred and eight patients (group A) received immediately a bedside ultrasound diagnostic test by expert investigastors at the time of ED admission, while 120 patients (group B) were evaluated and managed without a preliminary ultrasound diagnostic approach. The concordance between initial and final diagnosis was statistically significant in group $\mathrm{A} v s$ group $\mathrm{B}(\mathrm{P}<0.01)$. In-hospital mortality was significantly lower in group $\mathrm{A}$ as compared with group B [3 (2.7\%) vs 6 (5\%), respectively; $\mathrm{P}<0.01]$; in group $\mathrm{A}$ only nine patients $(8.3 \%)$ compared with seventeen patients $(14.1 \%)$ in group $\mathrm{B}(\mathrm{P}<0.01)$ were transferred to the intensive care unit for monitoring and treatment. The study proposed is not able to recommend the procedure because it is a retrospective design. In spite of this, our study supports the routine use of ultrasonography for the evaluation of patients having acute respiratory failure.

\section{Introduction}

Acute respiratory failure is one of the most distressing and more frequent conditions for patients in emergency departments (EDs). The immediate and accurate diagnosis is always imperfect and this sometimes compromises the patients' outcome. ${ }^{1-5}$

Ultrasound has long shown its utility for screening organs. ${ }^{6}$ Currently, the integrated ultrasound evaluation in the acute setting is

\section{Materials and Methods}

The present observational and retrospective study was conducted in the EDs of two hospitals in Como (Sant'Anna Hospital and Valduce Hospital). From January 2009 to December 2010, 256 consecutive adult patients admitted to the ED with acute respiratory failure were enrolled. Twenty-eight patients were excluded due their prompt intubation $(n=17)$ or absence of final diagnosis $(n=11)$. A total of 228 patients were evaluated for the study: 108 patients (group A) received a bedside ultrasound at admission by expert investigators, while 120 patients (group B) were managed without the ultrasound approach, due its prompt unavailability or the absence of a sonographer at admission. The competence of 11 out of 20 emergency physician ultrasonographers was demonstrated through multiple steps. Initially, they underwent a training course of fast echography in emergency setting and then they performed at least 100 noncardiac and 200 cardiac ultrasounds with a credentialed supervision. In addition, before the start of this study, all emergency physicians were given a course focusing on echocardiography.

The institutional review board of the two hospitals evaluated the study protocol and declared that the need for informed consent was waived.

\section{Patients}

The inclusion criteria were the following: age $>18$ years, signs of acute or acute on chronic respiratory failure, acute onset of severe respiratory distress (breathing rate $>35$ breaths/min), peripheral arterial oxygen saturation $\left(\mathrm{SpO}_{2}\right)<88 \%$, and breathing air $<90 \%$ with inhaled oxygen.

The patients excluded from the study were the following: i) patients immediately intubated and transferred to the intensive care unit
Correspondence: Andrea Bellone, Emergency Department, Sant'Anna Hospital, via Ravona 1, 22020 San Fermo della Battaglia, Italy.

Tel. +39.02.48703668 - Fax: +39.031.5855853

E-mail: andreabellone@libero.it

Key words: ultrasound, emergency, acute respiratory failure, diagnosis, outcome.

Contributions: $\mathrm{AB}$ and $\mathrm{ME}$ contributed to study design, subject enrollment, measurements, data management and analysis, and manuscript preparation. All other authors contributed to subject enrollment and manuscript preparation.

Conflict of interests: the authors declare no potential conflict of interests.

Conference presentation: the present paper was orally presented at the Italian Congress of the Academy of Emergency Medicine and Care (AcEMC) held in Rome on 2 November 2011 and it won the first prize.

Received for publication: 15 February 2013.

Revision received: 9 April 2013.

Accepted for publication: 11 April 2013.

This work is licensed under a Creative Commons Attribution 3.0 License (by-nc 3.0).

(C) Copyright A. Bellone et al., 2013

Licensee PAGEPress, Italy

Emergency Care Journal 2013; 9:e2

doi:10.4081/ecj.2013.e2

(ICU) if they presented acute dyspnea, respiratory distress with Glasgow coma scale $<13$, respiratory arrest, $\mathrm{SpO}_{2}<88 \%$ [or arterial oxygen tension $(\mathrm{PaO} 2)<55 \mathrm{mmHg}$ ] despite inspired oxygen fraction $>50 \%$, systolic arterial blood pressure (BP) $<90 \mathrm{mmHg}$, and signs of peripheral hypoperfusion; ii) patients presented with acute myocardial infarction signs or requiring dialysis for renal insufficiency.

Conversely, patients who presentend acute dyspnea and/or respiratory distress, and/or $\mathrm{SpO}_{2}<88 \%$ during oxygen therapy, and/or a worsening in level of consciousness after $2 \mathrm{~h}$ of optimal treatment - thus meeting the criteria of the hospital protocol for admitting patients from ED to ICU - were transferred to the ICU or to the intermediate care unit for monitoring and treatment and were part of the study. All the patients who received the ultrasound examination at admission belonged to group A. If ultrasound was not available, the patients were managed traditionally with clinical evaluation and chest x-ray and they were assigned to group B. The first diagnosis was made at the time of moving patients from the ED to either the ICU or the intermediate care unit or the respiratory/cardiology/general medicine division in both group of patients. The final diagnosis was made at the end of in-hos- 
pital patients' course on the basis of the hospital protocols.

\section{Ultrasound approach}

Ultrasound was performed using a machine (Vivid-I; General Electric Company, Fairfield, CT, USA) equipped with a 5-MHz microconvex probe. Patients were investigated in a semisupine position. The time spent for ultrasound evaluation at the time of ED admission was about $10 \mathrm{~min}$.

Ultrasound examination was made for a differential diagnosis among the most common causes of acute or acute on chronic respiratory failure [pulmonary edema, pulmonary embolism, pneumonia, acute exacerbation of chronic obstructive pulmonary disease (AECOPD) and acute asthma, pneumothorax and pericardial effusion] in order to manage patients adequately. We focused our attention on 8 items: i) prevalent A pattern (i.e. horizontal lines arising from the pleural line and found at regular intervals equalling the distance between the skin and the pleural line); ii) prevalent $B$ pattern (i.e. a hydroaeric comettail artefact arising from the pleural line and indicating interstitial syndrome); iii) lung sliding (i.e. visceral pleura sliding against the parietal pleura); iv) presence of alveolar consolidation and/or pleural effusion; v) qualitative diameter of right ventricle; vi) pericardial effusion; vii) inferior vena cava (IVC) collapsibility; and viii) femoral and/or poplitea venous compressibility. ${ }^{11-15}$

In group $\mathrm{A}$, the ultrasound evaluation together with clinical findings, electrocardiogram, arterial blood gases, venous blood samples, and chest x-ray data allowed an initial diagnosis that was compared with the final one. The same evaluation - except for the bedside ultrasound - was used for diagnosis and treatment in group B.

\section{Statistical analysis}

Physiologic data of patients are reported as means ( \pm standard deviation). Means are compared using the Student test. Fisher's exact test was employed to compare the rates of admission in ICU and in-hospital mortality in the two groups. The concordance between the first and the final diagnosis was analyzed using the $\chi^{2}$ test. P value $<0.05$ was considered statistically significant.

\section{Results}

This study included 256 patients admitted to the hospitals because of an acute respiratory failure between January 2009 to December 2010. Twenty-eight patients were excluded either because they were immediately intubated in ED and then transferred to ICU according to the hospital protocol ( $n=17)$, or because they did not received a definite final diagnosis $(n=11)$.

Two hundred and twenty-eight patients were analyzed for the study. According to the bedside ultrasound evaluation (group A), a prevalent bilateral B lines was observed in 52 cases (forty-eight were defined as pulmonary edema, while 4 patients were identified as affected by acute lung injury); while a predominant bilateral A lines was found in 28 cases (20 patients were defined as affected by AECOPD and/or acute asthma and 8 patients presented with an enlargement of the right ventricle diameter thus suggesting a diagnosis of pulmonary embolism) (5 of these patients the ultrasound veins compressibility test was positive). Twenty patients presented with an alveolar consolidation/pleural effusion. Five patients presented with an abolished lung sliding with predominant A lines (pneumothorax). Pericardial effusion was seen in 3 patients. Ultrasound was possible in all patients (feasi-

\section{bility of 100\%).}

In group B, 67 patients were initially defined as affected by pulmonary edema, while 12 patients were identified as affected by pneumonia/acute lung injury. Twenty patients were defined as affected by AECOPD and/or acute asthma, 11 by pulmonary embolism, and 6 patients by pneumothorax. Pericardial effusion was diagnosed in 4 patients. The diagnosis of patients belonging to group B was done by the emergency physicians on the basis of the clinical, laboratory and chest $\mathrm{x}$-ray.

Patients were similar in terms of age, sex, arterial blood gases, arterial BP, heart and respiratory rate, and acute physiologic chronic health evaluation II (APACHE II). Furthermore, comorbilities were not significantly different (Table 1). In-hospital mortality was significantly lower in group A as compared with group B [3 (2.7\%) vs $6(5 \%), \mathrm{P}<0.01]$. Only 9 patients of group A (8.3\%) vs 17 patients (14.1\%) of group B $(\mathrm{P}<0.01)$, were transferred to the ICU for monitoring and treatment (Table 2).

Table 1. Physiologic measurements and comorbilities of patients.

\begin{tabular}{lccc} 
& $\begin{array}{c}\text { Group A } \\
(\mathrm{n}=108)^{\circ}\end{array}$ & $\begin{array}{c}\text { Group B } \\
(\mathrm{n}=120)^{\circ}\end{array}$ & P value \\
Age (years) & $74.7 \pm 6.7$ & $76.1 \pm 5.7$ & $\mathrm{~ns}$ \\
Male/female ratio & $69 / 108$ & $75 / 120$ & $\mathrm{~ns}$ \\
\hline $\mathrm{pH}$ & $7.30 \pm 0.16$ & $7.31 \pm 0.18$ & $\mathrm{~ns}$ \\
$\mathrm{PaO2/FiO2} \mathrm{ratio}$ & $173 \pm 85$ & $177 \pm 96$ & $\mathrm{~ns}$ \\
\hline PaCO2 (mmHg) & $54 \pm 29$ & $53 \pm 31$ & $\mathrm{~ns}$ \\
Bicarbonate (mEq) & $23 \pm 8$ & $24 \pm 9$ & $\mathrm{~ns}$ \\
\hline Respiratory frequency (breaths/min) & $37 \pm 18$ & $38 \pm 22$ & $\mathrm{~ns}$ \\
Heart rate (beats/min) & $98 \pm 30$ & $103 \pm 33$ & $\mathrm{~ns}$ \\
\hline Systolic BP (mmHg) & $116 \pm 41$ & $112 \pm 35$ & $\mathrm{~ns}$ \\
Diastolic BP (mmHg) & $78 \pm 23$ & $68 \pm 27$ & $\mathrm{~ns}$ \\
\hline APACHE II score & $18.4 \pm 2.6$ & $18.9 \pm 4.6$ & $\mathrm{~ns}$ \\
Chronic heart failure (n) & $31 \pm 48$ & $35 \pm 51$ & $\mathrm{~ns}$ \\
\hline Arterial hypertension (n) & $59 \pm 46$ & $54 \pm 42$ & $\mathrm{~ns}$ \\
Diabetes (n) & $23 \pm 21$ & $26 \pm 19$ & $\mathrm{~ns}$ \\
\hline Atrial fibrillation (n) & $9 \pm 14$ & $12 \pm 18$ & $\mathrm{~ns}$ \\
Chronic renal failure (n) & $9 \pm \pm 15$ & $6 \pm 18$ & $\mathrm{~ns}$ \\
\hline Ischemic heart disease (n) & $26 \pm 50$ & $24 \pm 43$ & $\mathrm{~ns}$ \\
\hline ns, not significant; PaO2, arterial oxygen tension; Fi02, inspiratory oxygen fraction; PaCO2, arterial carbon dioxide tension; BP, blood pres- \\
sure; APACHE II, acute physiologic chronic health evaluation II. ${ }^{\circ}$ Values are expressed as means \pm standard deviation. &
\end{tabular}

Table 2. Patients' outcomes.

\begin{tabular}{lccc} 
& $\begin{array}{c}\text { Group A } \\
(n=108)\end{array}$ & $\begin{array}{c}\text { Group B } \\
(n=120)\end{array}$ & P value \\
\hline Admission to ICU - no. (\%) & $9(8.3)$ & $17(14.1)$ & 0.01 \\
In-hospital death - no. (\%) & $3(2.7)$ & $6(5)$ & 0.01 \\
\hline
\end{tabular}

$\mathrm{ICU}$, intensive care unit. 
Table 3. Comprehensive results: first and final diagnosis of the two groups.

\begin{tabular}{|c|c|c|c|c|c|c|c|}
\hline Group & Diagnosis & ACPE & AECOPD & Pneumothorax & $\begin{array}{c}\text { Pathology } \\
\text { Pneumonia-AL-ARDS }\end{array}$ & Pulmonary embolism & Pericardial effusion \\
\hline$A(n=108)$ & $\begin{array}{l}\text { First } \\
\text { Final }\end{array}$ & $\begin{array}{l}48 \\
44\end{array}$ & $\begin{array}{l}20 \\
23\end{array}$ & $\begin{array}{l}5 \\
5\end{array}$ & $\begin{array}{l}24 \\
26\end{array}$ & $\begin{array}{l}8 \\
7\end{array}$ & $\begin{array}{l}3 \\
3\end{array}$ \\
\hline$B(n=120)$ & $\begin{array}{l}\text { First } \\
\text { Final }\end{array}$ & $\begin{array}{l}67 \\
46\end{array}$ & $\begin{array}{l}20 \\
29\end{array}$ & $\begin{array}{l}6 \\
5\end{array}$ & $\begin{array}{l}12 \\
29\end{array}$ & $\begin{array}{c}11 \\
8\end{array}$ & $\begin{array}{l}4 \\
3\end{array}$ \\
\hline
\end{tabular}

ACPE, acute cardiogenic pulmonary edema; AECOPD, acute exacerbation of chronic obstructive pulmonary disease; ALI-ARDS, acute lung injury-acute respiratory distress syndrome.

Finally, the concordance between the initial and final diagnosis was significantly superior in group $\mathrm{A}$ in comparison with group $\mathrm{B}$ $(\mathrm{P}<0.01)$ (Tables 3 and 4).

\section{Discussion}

Bedside ultrasound in the EDs as a diagnostic approach to the acute respiratory failure resulted to be very useful and favorable in terms of in-hospital mortality and the need of intensive care assistance. In addition, the concordance between initial and final diagnosis was significantly in favour of patients submitted to the early ultrasound evaluation.

It is well known that lung and cardiac ultrasound immediately provide diagnosis of acute respiratory failure in $90.5 \%$ of cases. ${ }^{8} \mathrm{~A}$ recent review reported that ultrasonography is more accurate than auscultation or chest radiography for the detection of pleural effusion, consolidation, and alveolar interstitial syndrome in the critical care setting. ${ }^{16}$

No previous studies have analyzed the relationship between the bedside ultrasound approach and the patients' outcome. Only one previous study has shown that incorporating a goal-directed ultrasound protocol in nontraumatic, symptomatic hypotensive patients results in a more accurate physician impression of final diagnosis. ${ }^{17}$ The present retrospective study evaluated patients with acute respiratory failure from different causes. Results showed that the diagnosis of acute pulmonary edema is overestimated when the early bedside ultrasound is lacking. The possible explanation is that the clinical signs of acute pulmonary edema are often less specific, therefore the differential diagnosis is often complex. Moreover, it is possible to hypothesize an added value of saving time and resources when using ultrasound in severely dyspneic patients where the percent of misdiagnosis is about $20 \%$ avoiding a wrong and sometimes dangerous treatment. ${ }^{18,19}$ In our opinion, lung and cardiac ultrasound is an opportunity for those physicians who practice emergency medicine. Indeed, ultrasound,

Table 4. Concordance between first and final diagnosis of the two groups.

\begin{tabular}{lcccc} 
& Positive diagnosis & $\begin{array}{c}\text { Final diagnosis } \\
\text { Negative diagnosis }\end{array}$ & Total & P value \\
Group A & 98 & 10 & 108 & - \\
Group B & 68 & 52 & 120 & - \\
\hline Total & 166 & 62 & 228 & - \\
Group A vs group B & - & - & - & $<0.01$ \\
\hline
\end{tabular}

together with IVC collapsibility estimation, improves the possibility to perform a correct differential diagnosis and an appropiate management. It is known that the perception about lung ultrasound learning is a difficult exercise. Viceversa, appreciating lung sliding, B lines or IVC collapsibility has an extremely short learning curve. ${ }^{12,20}$

The relevance of our study was the evidence of a relationship between the bedside ultrasound approach and the prognosis of these patients. This result needs further studies to confirm the impact of diagnostic approach and the outcome of patients with acute respiratory failure.

Lastly, this study had some limitations. Most importantly, limitations include a retrospective design, the lack of uniformity between groups of treating physicians and a lack of analysis of what specific interventions or other factors could have accounted for the mortality benefit observed for patients in the ultrasound group. Second, the investigators in this study who have performed a bedside ultrasound evaluation were not blinded to the patient's management but in our acute setting this was not fulfilled. Third, we included patients with final several official diagnoses.

\section{Conclusions}

In conclusion, the study proposed is not able to recommend the procedure because it is retrospective design. In spite of this, our study supports the routine use of ultrasonography for the evaluation of patients having acute respiratory failure.

\section{References}

1. Wasserman K. Dyspnea on exertion: is it the heart or the lung? JAMA-J Am Med Assoc 1982;248:2039-43.

2. Aronchick J, Epstein D, Gefter WB, et al. Evaluation of the chest radiograph in the emergency department patient. Emerg Med Clin N Am 1985;3:491-501.

3. Ray P, Birolleau S, Lefort Y, et al. Acute respiratory failure in the elderly: etiology, emergency diagnosis and prognosis. Crit Care 2006;10:R82.

4. Greenbaum DM, Marschall KE. The value of routine daily chest X-rays in intubated patients in the medical intensive care unit. Crit Care Med 1982;10:29-30.

5. Lichtenstein D, Goldstein G, Mourgeon E, et al. Comparative diagnostic performances of auscultation, chest radiography and lung ultrasonography in acute respiratory distress syndrome. Anesthesiology 2004; 100:9-15.

6. Dénier A. Les ultrasons, leur application au diagnostic. Press Méd 1946;22:307-8.

7. Irwin RS, Rippe JM. Intensive care medicine. 6th ed. Philadelphia, PA: Lippincott Williams \& Wilkins; 2008.

8. Lichtenstein DA, Meziere GA. Relevance of lung ultrasound in the diagnosis of acute respiratory failure: the blue protocol. Chest 2008;134:117-25.

9. Zanobetti M, Poggioni C, Pini R. Can chest ultrasonography replace standard chest radiography for evaluation of acute dyspnea in the ED? Chest 2011;139:1140-7. 
10. Reissig A, Copetti R, Kroegel C. Current role of emergency ultrasound of the chest. Crit Care Med 2011;39:839-45.

11. Lichtenstein DA, Meziere G, Lascols N, et al. Ultrasound diagnosis of occult pneumothorax. Crit Care Med 2005;33:1231-8.

12. Lichtenstein DA, Meziere G, Biderman, et al. The lung point: an ultrasound specific to pneumothorax. Intensive Care Med 2000;26:1434-40.

13. Alrajhi K, Woo MY, Vaillancourt C. Test characteristics of ultrasonography for the detection of pneumothorax: a systematic review and meta-analysis. Chest 2012;141:
703-8.

14. Lichtenstein DA, Lascols N, Meziere G, et al. Ultrasound diagnosis of alveolar consolidation in the critically ill. Intens Care Med 2004;30:276-81.

15. Lichtenstein DA. Ultrasound diagnosis of pulmonary edema. Rev Im Med 1994;6:5612.

16. Moore CL, Copel JA. Point-of-care ultrasonography. New Engl J Med 2011;364:74957.

17. Jones AE, Tayal VS, Sullivan DM, Kline JA. Randomized, controlled trial of immediate versus delayed goal-directed ultrasound to identify the cause of nontraumatic hypotension in emergency department patients. Crit Care Med 2004;32:1703-8.

18. Rutten B, Cramer MJ, Lammers JW, et al. Heart failure and chronic obstructive pulmonary disease: an ignored combination? Eur J Heart Fail 2006;8:706-11.

19. Kristensen MS. Ultrasonography in the management of the airway. Acta Anaesth Scand 2011;55:1155-73.

20. Lichtenstein D, Meziere G. Training of general ultrasound by the intensivist. Réan Urg 1988;7(Suppl. 1):s108 (abstr.). 